\title{
A developmental sensitive period for spike timing-dependent plasticity in the retinotectal projection
}

\author{
JenniferTsui, Neil Schwartz and Edward S. Ruthazer*
}

Montreal Neurological Institute, McGill University, Montreal, OC, Canada

\section{Edited by:}

Per Jesper Sjöström, University

College London, UK

Reviewed by:

Hollis Cline, The Scripps Research

Institute, USA

Mu-Ming Poo, University of California

at Berkeley, USA

Susan B. Udin, State University of

New York, Buffalo, USA

*Correspondence:

Edward S. Ruthazer, Montreal

Neurological Institute, McGill

University, 3801 University Street,

Montreal, QC, H3A 2B4, Canada.

e-mail: edward.ruthazer@mcgill.ca
The retinotectal projection in Xenopus laevis has been shown to exhibit correlation-based refinement of both anatomical and functional connectivity during development. Spike timingdependent plasticity (STDP) is an appealing experimental model for correlation-based synaptic plasticity because, in contrast to plasticity induction paradigms using tetanic stimulation or sustained postsynaptic depolarization, its induction protocol more closely resembles natural physiological activity. In Xenopus tadpoles, where anatomical remodeling has been reported throughout much of the life of the animal, in vivo retinotectal STDP has only been examined under a limited set of experimental conditions. Using perforated-patch recordings of retina-evoked EPSCs in tectal neurons, we confirmed that repeatedly driving a retinotectal EPSP 5-10 ms prior to inducing an action potential in the postsynaptic cell, reliably produced timing-dependent long-term potentiation (t-LTP) of the retinotectal synapse in young wild type tadpoles (stages 41-44). At these stages, retinotectal timing-dependent long-term depression (t-LTD) also could be induced by evoking an EPSP to arrive 5-10 ms after an action potential in the tectal cell. However, retinotectal STDP using this standard protocol was limited to a developmental sensitive period, as we were unable to induce t-LTP or t-LTD after stage 44. Surprisingly, this STDP protocol also failed to induce reliable STDP in albino tadpoles at the early ages when it was effective in wild type pigmented animals. Nonetheless, low-frequency flashes to the eye produced a robust NMDA receptor-dependent retinotectal LTD in stage 47 albino tadpoles, demonstrating that the retinotectal synapse can nonetheless be modified in these animals using different plasticity paradigms.

Keywords: synaptic plasticity, retinotectal, albino, visual system, $\mathbf{N}$-methyl- $d$-aspartate receptor, Xenopus laevis, development

\section{INTRODUCTION}

Patterned neural activity and early sensory experience profoundly impact the development of organized circuit connectivity and can dramatically modify receptive field properties in the developing nervous system (Katz and Shatz, 1996; Holtmaat and Svoboda, 2009). The developing visual system has served as a model system in which to investigate the influences of sensory experience on circuit formation (Ruthazer and Cline, 2004; Huberman et al., 2008). In particular, the retinotectal projection in Xenopus laevis tadpoles has been a powerful platform for these studies, as it is possible to carry out both time-lapse imaging of structural development and electrophysiological measurements of synaptic physiology and receptive field structure on single neurons in the intact animal.

Computational models of activity-dependent map formation have traditionally employed a learning rule in which synapses between two neurons are strengthened in proportion to the degree of correlation in their firing (Abbott and Nelson, 2000). Such learning rules are sometimes referred to as "Hebbian", in recognition of the Canadian psychologist Donald O. Hebb, who first formally proposed that when one neuron "repeatedly or persistently takes part in firing" another neuron, its connection to that cell should be strengthened (Hebb, 1949). While many Hebbian models simplify this concept to reflect correlation in the firing rates of two cells, Hebb's original formulation specifically considered temporal causality.
Spike timing-dependent plasticity (STDP) is an experimentally observed form of Hebbian synaptic plasticity in which the precise order of firing of the pre- and postsynaptic partners determines the direction of changes in synaptic efficacy. STDP not only constitutes a plasticity mechanism that satisfies Hebb's requirement for temporal causality by strengthening those synapses at which the presynaptic cell fires just prior to its postsynaptic partner, but also includes a convenient mechanism for weakening synapses in the case when the postsynaptic cell fires first. It has been described in various forms in many circuits (Levy and Steward, 1983; Bell et al., 1997; Magee and Johnston, 1997; Markram et al., 1997; Feldman, 2000; Sjostrom et al., 2001), and was demonstrated in vivo in the Xenopus retinotectal system by Zhang et al. (1998). These seminal retinotectal experiments have been followed up by an impressive series of studies demonstrating the ability of STDP to modify various receptive field properties in the immature visual system, including direction selectivity and receptive field shape (Tao et al., 2001; Zhou et al., 2003; Vislay-Meltzer et al., 2005; Mu and Poo, 2006).

In principle, STDP could also provide an elegant potential mechanism for mediating the activity-dependent refinement and maintenance of topographic organization in the retinotectal circuit, a process in which patterned neural activity and $N$-methyl-Daspartate receptors (NMDARs) as correlation detectors have been directly implicated (Cline and Constantine-Paton, 1989; Ruthazer 
et al., 2003; Dong et al., 2009). Retinotectal map development in Xenopus is a protracted process that continues even after metamorphosis (Gaze et al., 1974). On the other hand, Xenopus retinotectal STDP experiments to date have focused almost exclusively on the initial period of innervation from developmental stages $40-45$, a period during which time retinotectal axons are largely overlapping within the tectal neuropil and the emerging retinotopic map is barely detectable anatomically (Sakaguchi and Murphey, 1985; O'Rourke and Fraser, 1990). It had been unknown, however, whether the same STDP mechanisms could also drive retinotectal input modification at later stages, perhaps participating in the activity-dependent refinement and maintenance of the retinotopic map. In the present study, we confirm previous findings that correlated firing of pre- and postsynaptic neurons within a narrow spike-timing window leads to robust timing-dependent long-term potentiation (t-LTP) and depression ( $t$-LTD) in early stage tadpoles, but find that after stage 44 the same STDP protocols no longer caused long-lasting changes in synaptic efficacy. On the other hand, we demonstrate a protocol for retinotectal plasticity induction using patterned visual stimulation that is effective in older tadpoles, arguing for distinct activity-dependent plasticity mechanisms participating at different stages of Xenopus retinotectal development.

\section{MATERIAL AND METHODS IN VIVOELECTROPHYSIOLOGY PREPARATION}

Wild type and albino $X$. laevis tadpoles staged 41-47 according to criteria from Nieuwkoop and Faber (1956) were anesthetized in modified Barth's solution (MBS-H) containing 0.02\% MS222 (Sigma) for dissection. For retinal loose patch stimulation experiments, the lens was removed from one eye to expose the retina. The skin on the head was cut and the brain was opened along the midline for recording in the contralateral tectal lobe. For recording, the tadpole was fixed to a Sylgard insert in the recording chamber with insect pins. The tadpole was constantly perfused with fresh external solution, and all experiments were performed at the room temperature. Movement of red blood cells could be observed in healthy animals in the vessels of the tectum and was monitored throughout the experiment.

\section{STDP EXPERIMENTS}

Whole-cell perforated-patch recording was performed as previously described (Zhang et al., 1998). The external solution was composed of (in $\mathrm{mM}$ ): $\mathrm{NaCl}, 115 ; \mathrm{KCl}, 2$; HEPES, $10 ; \mathrm{CaCl}_{2}, 3 ; \mathrm{MgCl}_{2}$, 1.5; glucose, 10; glycine, 0.005 ( $\mathrm{pH} 7.3$ ). To paralyze the tadpole, the external solution also contained $2.5 \mathrm{mM}$ tubocurare (Sigma). Borosilicate glass micropipettes (Warner), with a resistance in the range of 4-7 M $\Omega$ were briefly dipped in internal solution, and then back-filled with amphotericin B $(250 \mu \mathrm{g} / \mathrm{ml}$, Calbiochem $)$ containing internal solution. The internal solution contained (in $\mathrm{mM}$ ): K-gluconate, $110 \mathrm{KCl}, 10$; $\mathrm{NaCl}, 5 ; \mathrm{MgCl}_{2}$, 1.5; EGTA, 0.5; HEPES, 20; ATP, 2; GTP, 0.3 (pH 7.3). 4-7 M 2 patch pipettes were also used for extracellular retinal stimulation except that they were filled with external solution. Cells in the rostral tectum were targeted for recording. Test pulses were applied every $30 \mathrm{~s}$ in voltage clamp. Measurements of monosynaptically driven EPSCs were made using response latency to define the input. In a single case (t-LTP at stage 47) two consistently separable inputs onto a single cell were both included for analysis. STDP was induced by switching to current clamp mode and pairing retinal stimulation with postsynaptic current injection to produce an action potential in the tectal cell at the time intervals indicated. These pairings were repeated 100 times at $1 \mathrm{~Hz}$. Recordings were acquired with a patch clamp amplifier (Axopatch 200B; Axon Instruments) and Clampex software (Axon Instruments). Input resistance (0.5-1 G $\Omega$ ) and series resistance (30-70 M $\Omega$ ) were monitored continuously during recordings. Data were accepted for analysis only if the series resistance remained relatively constant $(<20 \%$ change) throughout the experiment. Cells were held at a constant potential of $-60 \mathrm{mV}$ (except during spike pairing). Liquid junction potential was not corrected.

\section{VISUALLY INDUCED LTD EXPERIMENTS}

Stage 47 albino tadpoles were immobilized while anesthetized $0.02 \%$ MS222 (Sigma) in MBS-H. A custom built harp was placed over the tadpole and the preparation was fixed in place in the recording chamber with 3\% low-melting point agarose and insect pins (Sigma). A window over the brain was then opened in the set agarose. The chamber was then flooded with external solution (in $\mathrm{mM}$ ): $135 \mathrm{NaCl}, 2 \mathrm{KCl}, 10 \mathrm{HEPES}, 10$ glucose, $3 \mathrm{CaCl}_{2}, 1.5 \mathrm{MgCl}_{2}$, pH 7.3, osm: 255-260. The skin was then split along the midline of the brain and the overlying pia mater carefully removed with a broken patch pipette to gain access to the tectal cells. A custom bent bipolar electrode (FHC) was then inserted into the optic chiasm. After allowing the preparation to stabilize for $20 \mathrm{~min}$, tectal cells were patched in the whole cell configuration using borosilicate glass pipettes with resistances of 4-9 M 2 . Access resistance (typically $50 \mathrm{M} \Omega$ ) was monitored throughout the experiment and cells that changed by more than $20 \%$ were excluded. The internal solution consisted of $120 \mathrm{~K}$-gluconate, $5 \mathrm{NaCl}, 1.5 \mathrm{MgCl}, 20 \mathrm{HEPES}$, 1 EGTA, 2 ATP, 0.3 GTP (in mM). For monitoring-evoked currents, cells were clamped at $-70 \mathrm{mV}$. Cells were discarded if evoked events had latencies that varied with stimulus intensity, if the event did not occur within $5 \mathrm{~ms}$ of the stimulus, or if more than two failures were observed during the baseline. After a $6 \mathrm{~min}$ baseline (test stimulus every $30 \mathrm{~s}$ ), the recording was switched to current clamp $(I=0$; average resting potential $-67.4 \mathrm{mV})$. The animal was then stimulated with a $4 \times 3$ array of green LEDs placed approximately $12 \mathrm{~cm}$ from the eye contralateral to the recording pipette for $15 \mathrm{~min}$, with each of the four rows of LEDs illuminating for $1 \mathrm{~s}$ in sequence followed by $1 \mathrm{~s}$ of darkness $(1 \mathrm{~Hz}$ transitions within a $0.2 \mathrm{~Hz}$ cycle). Cells exhibited consistent subthreshold response to the LEDs but did not spike consistently in response to the stimulus. After the 15 min stimulation period, EPSCs were again monitored at $-70 \mathrm{mV}$ in voltage clamp. For NMDA receptor blockade, CPP $(40 \mu \mathrm{m})$ (Tocris) was bath applied $1 \mathrm{~min}$ after obtaining the whole cell configuration.

\section{DATA ANALYSIS}

Data were analyzed using ClampFit software (Molecular Devices). Statistics were performed with SPSS software. Plasticity was assessed using a one-sample $t$-test on EPSC peak amplitudes averaged from 20 to $30 \mathrm{~min}$ after induction, normalized to the baseline amplitudes collected during the 10 minutes immediately before 
induction. Differences across experimental groups were analyzed for significance using Student's $t$-test for two groups and ANOVA with Bonferroni post hoc analyses for multiple groups.

\section{EXPERIMENTAL ANIMALS}

Experiments were approved by the Montreal Neurological Institute Animal Research Committee and performed in accordance with guidelines of the Canadian Council on Animal Care.

\section{RESULTS}

\section{STDP INDUCTION IN THE XENOPUS TADPOLE}

We sought to determine whether retinotectal STDP mechanisms that have been implicated in early retinotectal plasticity and receptive field modifications continue to be expressed at later stages as the anatomical retinotectal map gradually emerges and tectal neuronal receptive fields refine (O'Rourke and Fraser, 1990; Tao and Poo, 2005). In vivo perforated-patch voltage clamp recordings were made to monitor the strength of retinal ganglion cell (RGC) synaptic inputs over time (Figure 1A; Zhang et al., 1998). Extracellular stimulation using a loose patch electrode positioned in the contralateral eye evoked a monosynaptic inward current with an onset latency of about $5 \mathrm{~ms}$ in tectal cells clamped at $-60 \mathrm{mV}$ to block NMDARs (Figure 1B). The amplitude of this evoked excitatory postsynaptic current (EPSC) was monitored every $30 \mathrm{~s}$ to measure changes in retinotectal synaptic efficacy.

For the induction of STDP, recordings were temporarily switched to current clamp mode to permit the firing of an action potential in response to depolarizing current injection through the recording pipette. One of three standard induction protocols was applied: (1) for t-LTP, retinal stimulation was timed to evoke an EPSP 5-10 ms prior to the induction of an action potential in the tectal neuron (Figure 1C), (2) for t-LTD the EPSP was timed to arrive 5-10 ms after the action potential (Figure 1D), and (3) a negative control protocol in which the interval between the action potential and the EPSP is outside the window to induce synaptic plasticity (+100 ms interval, Figure 1E). Intervals of 5-10 ms were chosen because these timing differences produced large changes in synaptic efficacy in previous reports (Zhang et al., 1998). Pairings were repeated 100 times at $1 \mathrm{~Hz}$.

\section{RETINOTECTAL STDP IN WILD TYPE TADPOLES IS LIMITED TO EARLY DEVELOPMENT}

As illustrated in the example from a stage 43 tadpole, the pre/post pairing protocol produced a robust retinotectal t-LTP in young, stage 41-44 wild type tadpoles (Figure 2A) consistent with previous reports (Zhang et al., 1998). This protocol for induction of t-LTP was highly

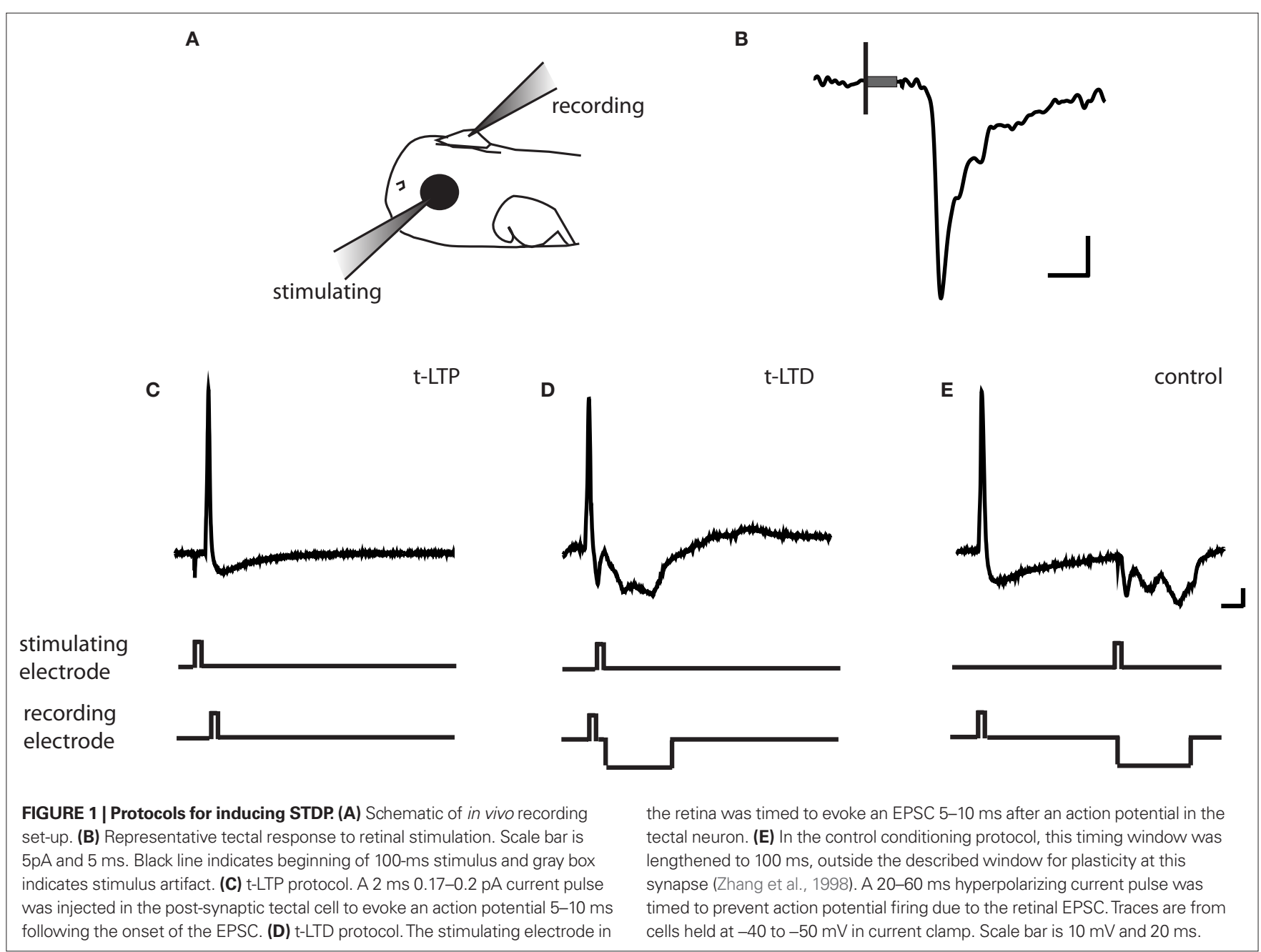




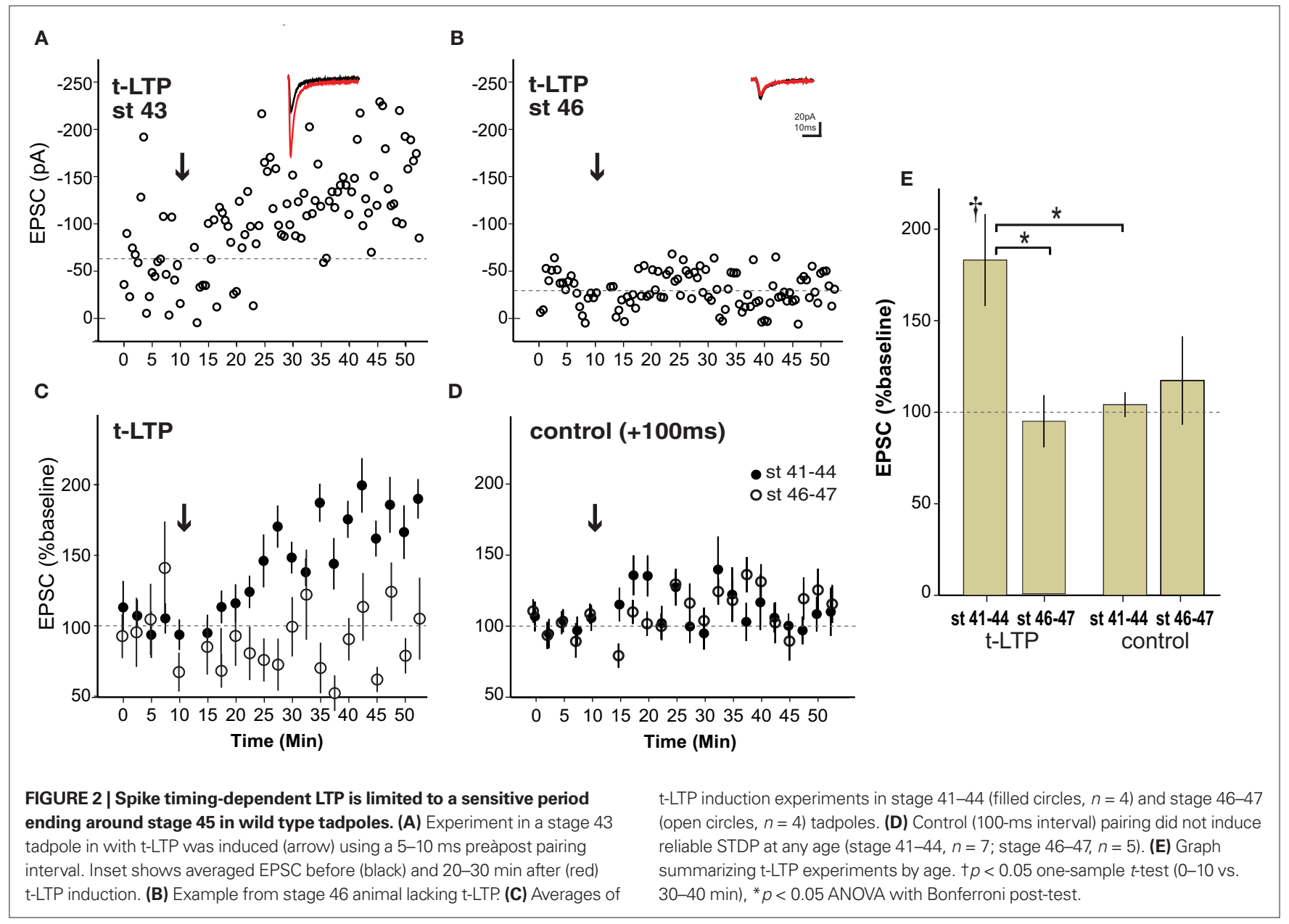

reliable, leading to a significant increase in EPSC amplitude by $20 \mathrm{~min}$ after induction $(183 \pm 25 \%$ baseline, $p<0.05)$ in four out of four cases (Figure 2C). In contrast, the same induction protocol failed to produce a potentiation ( $94 \pm 14 \%$ baseline, $n=4, p>0.05$ ) in animals after stage 45 (Figures 2B,C). The control pairing protocol (+100-ms interval) did not cause a significant change in EPSC amplitude at any age tested ( $108 \pm 10 \%$ baseline, $n=12, p>0.05$ ), confirming that the potentiation before stage 45 was indeed a consequence of the spike timing interval rather than a non-specific synaptic run-up following repeated stimulation in the younger animals (Figures 2D,E). This result demonstrates that t-LTP in wild type tadpoles is restricted to a developmental sensitive period that ends around stage 45 .

The mechanisms underlying t-LTP and t-LTD have been shown to be separable in some systems (Bender et al., 2006). We therefore examined whether retinotectal t-LTD was also restricted to a developmental period similar to that for t-LTP. Figure 3A shows the induction of t-LTD in a stage 41 tadpole, during the sensitive period. On the other hand at stage 45 t-LTD could not be induced using the same protocol (Figure 3B). Overall, the t-LTD induction protocol produced a significant reduction of EPSC amplitude in stage 41-44 wild type tadpoles ( $65 \pm 11 \%$ baseline, $n=7, p<0.05$; Figure $3 \mathrm{C}$ ). The same protocol in older tadpoles did not reliably produce t-LTD, but instead led to a small, non-significant increase in EPSC amplitude (123 $\pm 12 \%$ baseline, $n=6, p>0.05)$. Thus, retinotectal t-LTD also exhibits an age-dependence with tadpoles younger than stage 45 responding differently from older animals (stage $41-44$ vs. stage $45-47, p<0.01$; Figure 3D). Figure 4 presents a scatter plot of all the STDP experiments by age. While it is not possible from this dataset to precisely define an exact point when the STDP sensitive period ends it is clear that the efficacy of the standard spike-timing protocols to induce reliable synaptic changes falls off substantially around stage 45 in wild type tadpoles.

\section{ALBINO TADPOLES DO NOT EXHIBIT ROBUST CLASSIC RETINOTECTAL STDP}

The albino $X$. laevis tadpole is a useful experimental model for in vivo imaging due to the relative ease with which the brain can be visualized in the intact animal. In order to be able to more directly relate retinotectal imaging data from the literature to synaptic plasticity studies, we examined STDP in albino tadpoles (Figure 5). Surprisingly, neither t-LTP ( $97 \pm 28 \%$ baseline, $n=4)$ nor t-LTD ( $96 \pm 7 \%$ baseline, $n=4$ ) could be reliably induced in albino tadpoles during the STDP sensitive period defined from wild type animals.

\section{VISUAL STIMULATION PROTOCOL CAN INDUCE RETINOTECTAL LTD IN STAGE 47 ALBINO TADPOLES}

Previous experiments in albino tadpoles in which signaling through $\mathrm{Ca} /$ calmodulin kinase 2 (Wu et al., 1996), AMPAR trafficking (Haas et al., 2006) or calcineurin (Schwartz et al., 2009) 
A
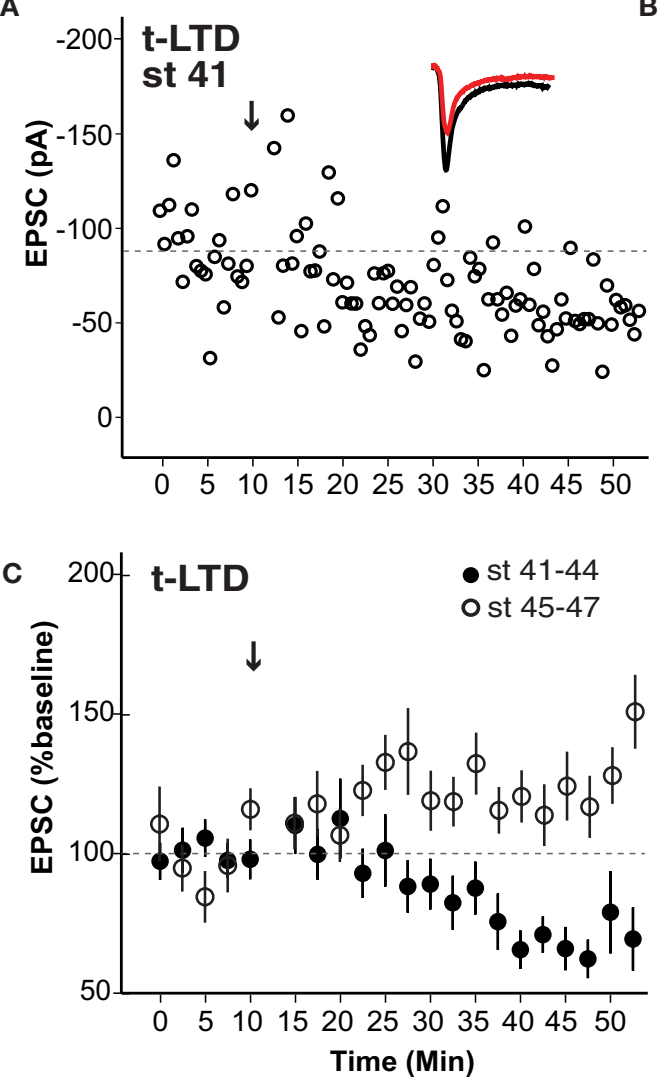
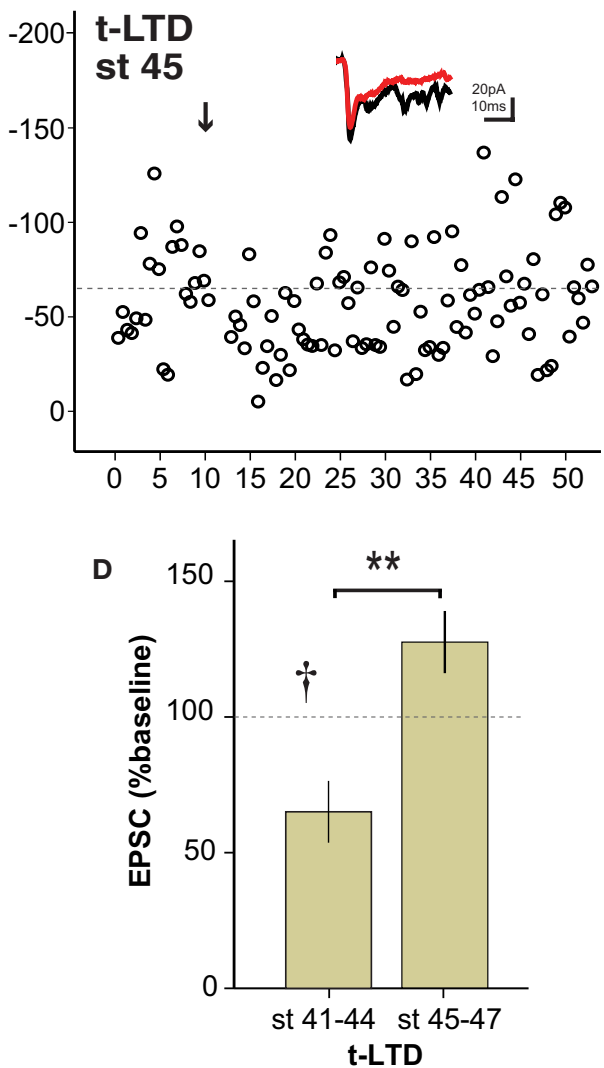

FIGURE 3 |The sensitive period for spike timing-dependent LTD is similar to that for t-LTP. (A) Experiment from a stage 41 tadpole in which t-LTD was induced (arrow) using the 5-10 ms postàpre protocol. (B) The same pairing protocol failed to induce synaptic depression in a stage 45 tadpole. Insets show sample traces as in Figure 1. (C) Average of all t-LTD experiments grouped by developmental stage. (D) Graph summarizing t-LTD experiments by developmental stage. $n=7$ stage $41-44, n=6$ stage $45-47,+p<0.05$ one-sample t-test (0-10 vs. 30-40 min), ** $p<0.01$ Student's t-test. was genetically disrupted suggest that they are likely to possess the necessary signaling machinery to exhibit some forms of retinotectal synaptic plasticity. Furthermore, Dunfield and Haas (2009) have shown that repeated presentation of visual stimuli can induce a long-lasting change in the amplitude of calcium signals measured at tectal cell somata in stage 50 albino tadpoles. The limitation of such measurements is that they cannot distinguish between retinotectal synaptic plasticity and changes in the intrinsic excitability or local connectivity of the tectal neurons or retinal neurons themselves. We therefore examined the effects of repeated visual stimulation on retinotectal synaptic efficacy in albino tadpoles by placing a bipolar stimulating electrode in the optic chiasm to evoke test pulses in RGC afferent axons. Plasticity was induced by sequentially flashing an array of LEDs in front of the eye contralateral to the recording pipette at low frequency for $15 \mathrm{~min}$ (see Section "Methods"). This low-frequency visual stimulation protocol resulted in a dramatic LTD of the retinotectal EPSC amplitude ( $59 \pm 10 \%$ baseline, $n=9)$ in stage 47 albino tadpoles (Figures 6A,C). This LTD was significantly attenuated $(85 \pm 6 \%$ baseline, $n=4, p<0.001$ ) in the presence of the NMDAR antagonist CPP (Figures 6 B,C). These results demonstrate that the Xenopus retinotectal synapse can exhibit robust LTD in response to natural sensory stimulation in older albino animals despite the fact that neither albino nor late stage tadpoles appeared to exhibit STDP when tested using standard induction protocols.

\section{DISCUSSION}

Our study confirmed previous reports that repeated pairing of pre- and postsynaptic neuronal firing using a 5-10 ms EPSP-tospike interval is highly effective at driving retinotectal t-LTP and t-LTD in the developing Xenopus visual system. We found that STDP induced by this classic protocol is restricted to the period of early retinotectal development ending around stage 45 in wild type tadpoles. Surprisingly, unlike wild type pigmented animals, albino tadpoles did not exhibit reliable STDP even during the sensitive period. Stage 47 albino tadpoles nonetheless did show a profound retinotectal LTD in response to repeated low-frequency visual stimulation, suggesting that diverse mechanisms can participate in retinotectal circuit plasticity.

\section{SENSITIVE PERIODS IN CIRCUIT DEVELOPMENT}

Sensitive periods for developmental plasticity in many different brain areas have been described, including for ocular dominance shifts in primary visual cortex (Wiesel, 1982; Hensch, 2005), eye 


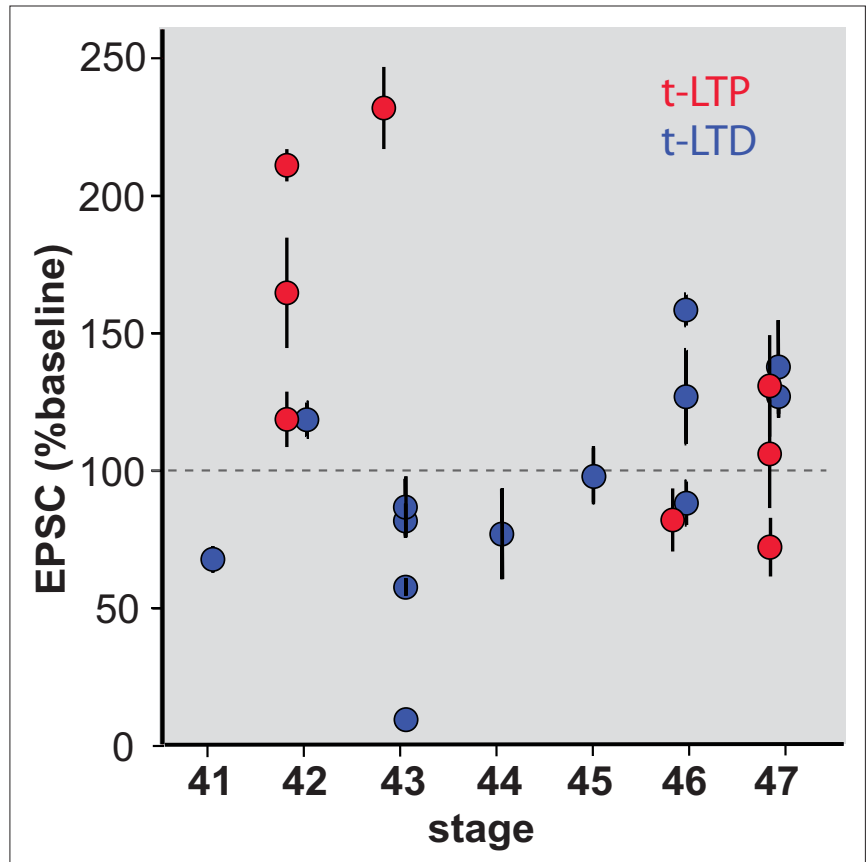

FIGURE 4 | Scatterplot showing STDP efficacy as a function of developmental stage. Mean EPSC amplitude measured at 20-30 min after induction, normalized to baseline amplitude during the $10 \mathrm{~min}$ immediately prior to induction.

specific segregation in the lateral geniculate nucleus (Huberman et al., 2008), thalamocortical LTP and LTD (Inan and Crair, 2007), and map plasticity in supragranular layers of cortex (Kirkwood et al., 1995; Li et al., 2009). While at first glance it may seem that any loss of potential for plasticity imposes undesirable limits on a developing circuit, it is likely that the development of fully functional circuits requires that the susceptibility of some inputs, especially primary inputs, to undergo plastic changes be restricted to permit the progressive refinement of others. For example, the relatively early stabilization of thalamic inputs to layer 4 of cortex may be necessary to provide the precise timing of firing in layer 4 that later drives STDP in the projection to layer 2/3 (Celikel et al., 2004). The mapping of auditory space onto the visuotopic map in the optic tectum in barn owls is another example where restricted plasticity in the visual inputs facilitates appropriate modifications of the coordinated auditory inputs (Knudsen, 2002). Similarly, in the Xenopus optic tectum STDP of recurrent excitation, which helps sculpt the temporal properties of the tectal response (Pratt et al., 2008), and multimodal integration in which other sensory modalities are mapped onto the retinotopic representation (Deeg et al., 2009; Hiramoto and Cline, 2009) are both likely to benefit from reduced plasticity in the primary sensory inputs from the retina. Interestingly, STDP of recurrent excitation in the tectum has been observed at stages $47-48$, meaning that distinct populations of synapses onto the same cells can exhibit different potential to undergo this form of plasticity.

Tectal growth, shifting of retinal terminals across the developing tectum and accompanying receptive field changes proceed long after the period when STDP can no longer be induced (Fraser, 1983; Sakaguchi and Murphey, 1985). Our finding that specific patterns of visual stimulation were still capable of driving retinotectal synaptic changes at a later stage of development raises the possibility that other plasticity mechanisms than STDP may be involved in refining tectal cell responses in older animals. STDP may be the mechanism best suited for implementing temporally precise interactions in the immature visual system while other mechanisms are more efficient at later stages. For example, STDP has been shown to be input specific from the youngest stages at which it has been examined in Xenopus, whereas induction of LTP by theta-burst stimulation (TBS) lacks tight input specificity in very immature tectal neurons, but becomes input specific at roughly the same stage that STDP can no longer be induced (Tao et al., 2001). Thus retinotectal STDP might serve as a transient means to refine the initial projection until it becomes mature enough to take full advantage of other mechanisms.

\section{MECHANISTIC CONSIDERATIONS}

Several potentially relevant developmental changes occur in tectal neurons around stage 45 that could underlie the loss of STDP. One important developmental shift that occurs around this stage, but continues much later, is the KCC2-mediated conversion of the chloride reversal potential $\left(\mathrm{E}_{\mathrm{Cl}}\right)$ from depolarizing to hyperpolarizing (Akerman and Cline, 2006). However, we do not believe that this change can fully explain our observations. First of all, despite the evidence that the gradual shift in $\mathrm{E}_{\mathrm{C}}$ is already under way, its reported value as late as stage 47 is still fairly depolarizing relative to the typical resting membrane potential of a tectal cell. Secondly, the retinotectal projection is exclusively glutamatergic and the vast majority of polysynaptic activation does not occur until after the STDP pairing period. Finally, we found that adding picrotoxin to the bath did not impact our ability to induce STDP (data not shown).

Another important event is that the lateral spread of stimulusevoked calcium influx within tectal neuron dendrites becomes spatially restricted around this time, possibly reflecting a change in the calcium buffering capacity of the cell (Tao et al., 2001). It has been proposed that STDP may depend in part on a non-linear summation of calcium elevation induced by the EPSP and the backpropagating action potential (Nevian and Sakmann, 2004). Thus, the development increase in the calcium buffering capacity of tectal neurons could potentially impact their ability to undergo STDP.

\section{OTHER PLASTICITY INDUCTION PROTOCOLS}

This is the first report of a sensitive period for retintotectal plasticity in Xenopus to our knowledge. However in the regenerating retinotectal system of goldfish, it has been reported that the period of greatest susceptibility to LTP, induced by $0.1 \mathrm{~Hz}$ supramaximal stimulation of the optic nerve, corresponds to a period from 20 to 40 days post-nerve-crush, when RGC inputs are still actively in the process of reestablishing contacts (Schmidt, 1990). One limitation of our current experimental design is that in order to test STDP at a range of developmental stages, we were forced to focus on just three spike-timing intervals, $+100 \mathrm{~ms}$ post $\rightarrow$ pre, $5-10 \mathrm{~ms}$ pre $\rightarrow$ post and $5-10 \mathrm{~ms}$ post $\rightarrow$ pre. It is conceivable, for example, that beyond the ages defined as the sensitive period in our experiments a shorter timing interval might have produced reliable plasticity. 
A
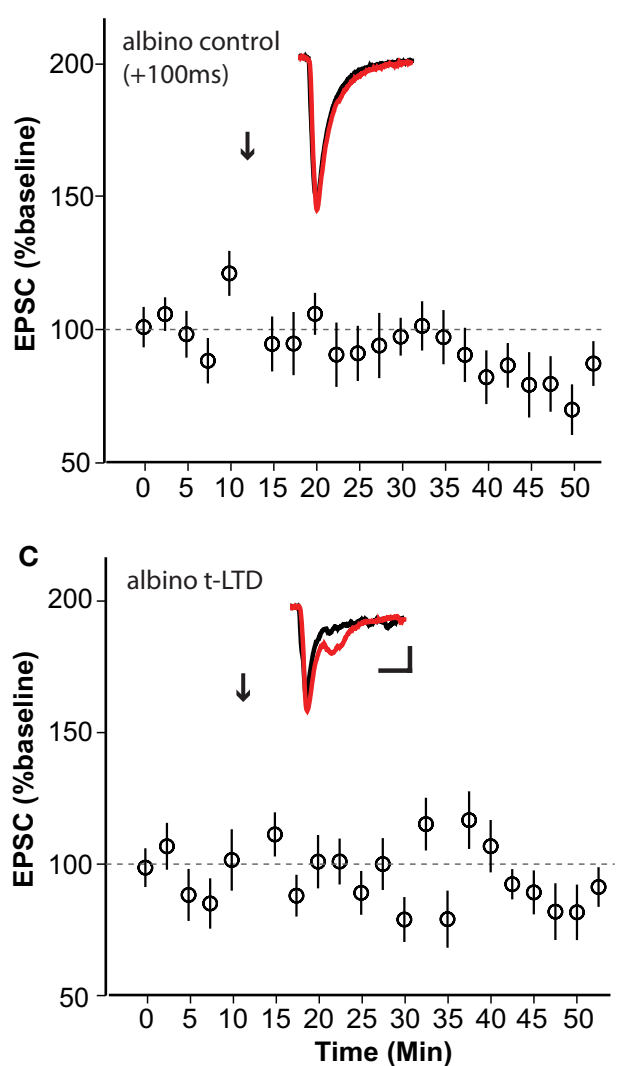

B

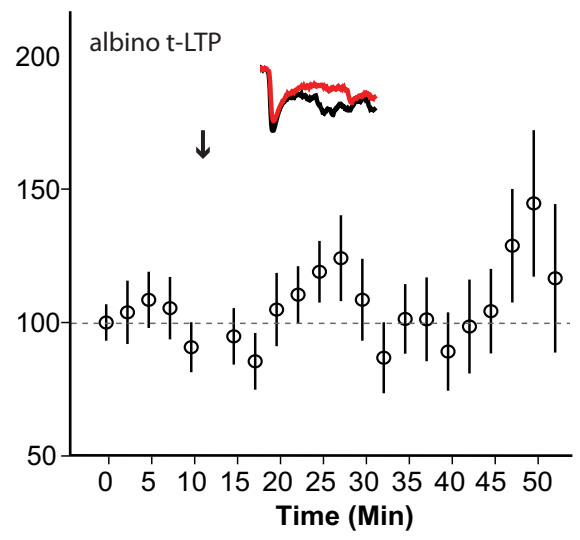

D

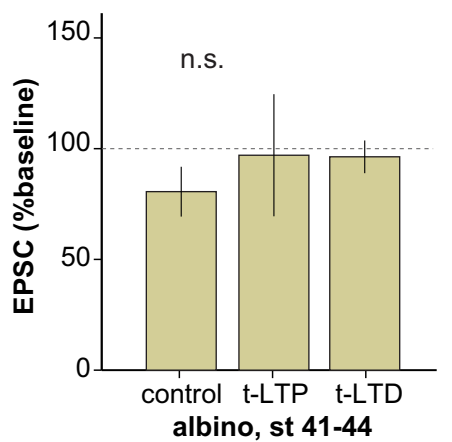

FIGURE 5 | Albino Xenopus tadpoles do not exhibit reliable STDP during the sensitive period. (A-C) Average of experiments to induce t-LTP ( $n=4)$ (B) and t-LTD $(n=4)(C)$ using the protocols that succeeded in wild type animals, applied to stage $41-44$ albino tadpoles. No STDP was reliably generated using the classic STDP protocol in albinos.

It remains unclear whether STDP and other synaptic plasticity induction protocols act through common biochemical pathways within tectal cells or depend on different signaling cascades. Tectal neurons are morphologically quite small and their high input resistances $(0.5-1 G \Omega)$ render them are electrotonically compact, making it unlikely that the loss of STDP in older animals was simply due to a failure of action potential back-propagation. Inhibition was not blocked in these experiments, raising the possibility of local dendritic shunting in older animals as a possible mechanism by which STDP might be lost with age (Corlew et al., 2007). However, in pilot experiments performed with picrotoxin in the bath, we were also unable to induce STDP in older tadpoles (data not shown) - these were discontinued, however, as excessive recurrent activity in picrotoxin interfered with accurate measurement of evoked EPSC amplitudes.

Although retinotectal STDP could not be induced after stage 45 , repeated visual stimulation was able to induce a robust NMDAR-dependent LTD at stage 47. Other groups have previously demonstrated retinotectal plasticity induced using slightly different visual stimulation protocols at younger (Zhang et al., 2000) and older (Dunfield and Haas, 2009) stages. What properties of visually induced plasticity might be permissive for effecting synaptic changes in older animals when classic STDP protocols fail? Unlike the STDP protocol in which only a small handful of inputs are activated during conditioning, visual induction of plasticity involves the coherent excitation of a large number of RGCs. In this case, for example, there may be sufficient convergent afferent drive to overcome a developmental change in calcium buffering of the neuron or whatever critical change may have led to the loss of STDP.

\section{LACK OF STDP IN ALBINO TADPOLES}

We found that STDP was not reliably induced in albino tadpoles during the wild type sensitive period. While it is possible that albino animals may simply have a delayed sensitive period, this would not be consistent with the fact that morphological and basic electrophysiological properties of RGCs and tectal neurons are overall indistinguishable between wild type and albino tadpoles of the same ages. The differences in STDP sensitivity between wild type and albino tadpoles can perhaps be explained by genetic differences between animals. Albinos of many species including humans, in which the tyrosinase gene is disrupted, are known to have abnormalities in the anatomy and physiology of their visual systems (Diykov et al., 2008; Herrera and Garcia-Frigola, 2008), however the Xenopus mutation does not involve this gene (MacMillan, 1979). Xenopus mutants exhibit periodic albinism, in 


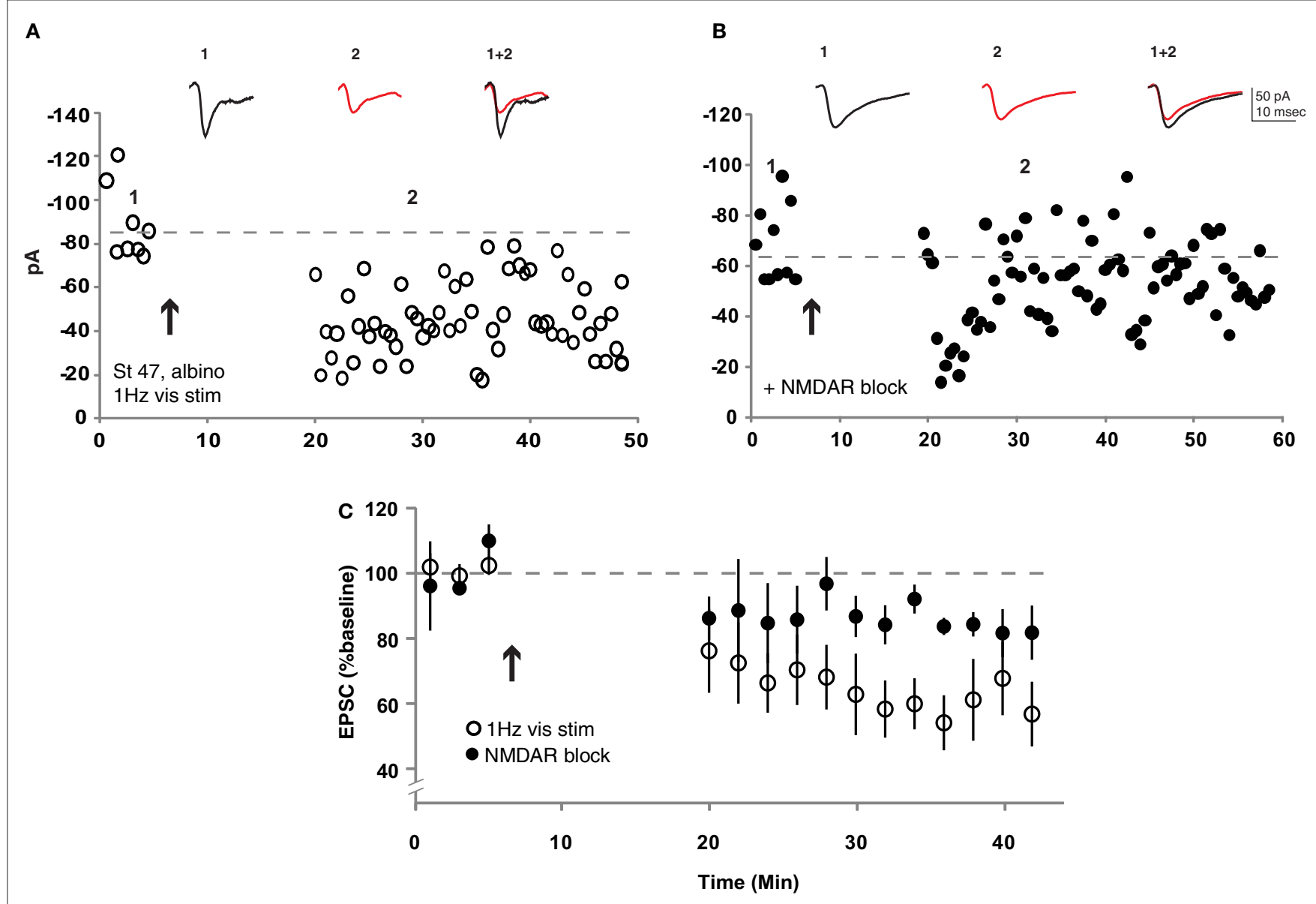

FIGURE 6 | Repeated low-frequency visual stimulation induced LTD in albino stage $\mathbf{4 7}$ tadpoles. (A) Repeatedly flashing an array of LEDs at $1 \mathrm{~Hz}$ for $15 \mathrm{~min}$ induced retinotectal synaptic depression. (B) This visual stimulation-induced LTD was largely blocked in the presence of CPP $(40 \mathrm{mM})$. (C) Average of all experiments with (filled circles, $n=4$ ) and without (open circles, $n=9$ ) NMDARs pharmacologically blocked. $p<0.001$ Student's $t$-test. which melanophore production occurs but is delayed and reduced. However, as the specific genes involved in the Xenopus albino mutation are not known, there could potentially be a subtle change in signaling or synaptic function that would alter the response to the STDP protocol.

Alternatively, differences in past sensory experience between albino and wild type tadpoles may account for their differential sensitivities. Although laboratory strains of albino tadpoles do not entirely lack melanophores, a striking feature of albino tadpoles is the extremely sparse pigmentation around their eyes, which in wild type animals is dark enough to completely block light from entering the retina except through the lens. This difference in normal visual input could potentially drive meta-plasticity such that albinos, which would have generally less well-structured activation of their photoreceptors may somehow lose sensitivity to precisely timed input. Dark-rearing of wild type and albino tadpoles to eliminate differences in visual experience might be informative in this case, but could be confounded by additional changes in the susceptibility of the visual system to undergo synaptic plasticity. A better experiment would be to take advantage of the ability to transplant tissues in tadpole embryos by examining STDP in wild type animals in which an eye primodium from an albino embryo had been transplanted to produce a chimera with a wild type tectum innervated by a faintly pigmented albino eye.

\section{FUTURE DIRECTIONS}

Given that retinotectal map remodeling occurs over an extended time beyond the STDP sensitive period, the specific contribution of STDP to retinotectal function and map refinement remains unclear. Does this relatively brief period of early plasticity provide a foundation upon which later tectal development occurs? It may be possible to exploit the differences between wild type and albino animals to determine electrophysiologically and anatomically whether their retinotopic organization differs immediately following the critical period and then several weeks afterwards when alternative plasticity mechanisms have had a chance to act in both. Ultimately, t-LTP and t-LTD protocols are merely idealized experimental assays that at best approximate natural sensory stimuli encountered during normal development. Testing retinotectal connectivity changes in response to presentation of natural scenes (Froemke and Dan, 2002) that closely mimic what tadpoles might see in the wild might provide the best understanding of how activity-dependent plasticity truly contributes to the development of visual processing. 


\section{ACKNOWLEDGMENTS}

The authors thank Dr. Carlos Aizenman for assistance in setting up the initial recordings. This work was supported by grants to Edward S. Ruthazer from the March of Dimes, EJLB Foundation, the Canadian Institutes of Health

\section{REFERENCES}

Abbott, L. F., and Nelson, S. B. (2000). Synaptic plasticity: taming the beast. Nat. Neurosci. 3, 1178-1183.

Akerman C. J., and Cline H. T. (2006). Depolarizing GABAergic conductances regulate the balance of excitation to inhibition in the developing retinotectal circuit in vivo. J. Neurosci. 26, 5117-5130.

Bell, C. C., Han, V. Z., Sugawara, Y., and Grant, K. (1997). Synaptic plasticity in a cerebellum-like structure depends on temporal order. Nature 387, 278-281.

Bender, V. A., Bender, K. J., Brasier, D. J., and Feldman, D. E. (2006). Two coincidence detectors for spike timingdependent plasticity in somatosensory cortex. J. Neurosci. 6, 4166-4177.

Celikel, T. Stostak, V. A., and Feldman, D. E. (2004). Modulation of spike timing by sensory deprivation during induction of cortical map plasticity. Nat. Neurosci. 7, 534-541.

Cline, H. T., and Constantine-Paton, M. (1989). NMDA receptor antagonists disrupt the retinotectal topographic map. Neuron 3, 413-426.

Corlew, R., Wang, Y. Ghermazien, H., Erisir, A., and Philpot, B. D. (2007). Developmental switch in the contribution of presynaptic and postsynaptic NMDA receptors to long-term depression. J. Neurosci. 27, 9835-9845.

Deeg, K. E., Sears, I. B., and Aizenman, C. D. (2009). Development of multisensory convergence in the Xenopus optic tectum. J. Neurophysiol. 102, 3392-3404.

Dong W., Lee, R. H., Xu, H., Yang, S., Pratt, K. G., Cao, V., Song, Y. K., Nurmikko, A., and Aizenman CD. (2009). Visual avoidance in Xenopus tadpoles is correlated with the maturation of visual responses in the optic tectum. J. Neurophysiol. 101, 803-815.

Diykov, D. Turchinovich, A. Zoidl, G., and Hofmann, K. P. (2008). Elevated intracellular chloride level in albino visual cortex neurons is mediated by $\mathrm{Na}-\mathrm{K}-\mathrm{Cl}$ co-transporter. $B M C$ Neurosci. 9, 57.

Dunfield, D., and Haas, K. (2009). Metaplasticity governs natural experience-driven plasticity of nascent embryonic brain circuits. Neuron 64 , 240-250.

Feldman, D. E. (2000). Timing-based LTP and LTD at vertical inputs to layer II/
III pyramidal cells in rat barrel cortex. Neuron 27, 45-56.

Fraser, S.E. (1983). Fiber optic mapping of the Xenopus visual system: shift in the retinotectal projection during development. Dev. Biol. 95, 505-511.

Froemke, R. C., and Dan,Y.(2002).Spiketiming-dependent synaptic modification induced by natural spike trains. Nature 416, 433-438.

Gaze, R. M., Keating, M. J., and Chung, S. H. (1974). The evolution of the retinotectal map during development in Xenopus. Proc. R. Soc. Lond. B Biol. Sci. 185, 301-330.

Haas, K. Li, J., and Cline, H. T. (2006). AMPA receptors regulate experiencedependent dendritic arbor growth in vivo. Proc. Natl. Acad. Sci. USA 103, 12127-12131.

Hebb, D. O. (1949). The Organization of Behavior: A Neuropsychological Theory. New York: Wiley.

Hensch, T. K. (2005). Critical period plasticity in local cortical circuits. Nat. Rev. Neurosci. 6, 877-888

Herrera, E., and Garcia-Frigola, C. (2008) Genetics and the development of the optic chiasm. Front. Biosci. 13, 1646-1653.

Hiramoto, M., and Cline, H. T. (2009). Convergence of multisensory inputs in Xenopus tadpole tectum. Dev. Neurobiol. 69, 959-971.

Holtmaat, A., and Svoboda, K. (2009). Experience-dependent structural synaptic plasticity in the mammalian brain. Nat. Rev. Neurosci. 10 647-658.

Huberman, A. D., Feller, M. B., and Chapman, B. (2008). Mechanisms underlying development of visual maps and receptive fields. Annu. Rev. Neurosci. 31, 479-509.

Inan, M., and Crair, M. C. (2007) Development of cortical maps: perspectives from the barrel cortex. Neuroscientist 13, 49-61.

Katz, L. C., and Shatz, C. J. (1996). Synaptic activity and the construction of cortical circuits. Science 274, 1133-1138.

Kirkwood, A., Lee H. K., and Bear, M. F. (1995). Co-regulation of long-term potentiation and experience-dependent synaptic plasticity in visual cortex by age and experience. Nature $375,328-331$

Knudsen, E. I. (2002). Instructed learning in the auditory localization pathway of the barn owl. Nature 417, 322-328.

Research, and by fellowships from the following agencies: MNI Jeanne-Timmins-Costello (Jennifer Tsui), Fonds de la recherche en santé Québec (Jennifer Tsui), and IPN JeanneTimmins-Costello (Neil Schwartz), Scottish Rite Charitable Foundation (Neil Schwartz).

Levy, W. B., and Steward, O. (1983). Temporal contiguity requirements for long-term associative potentiation/depression in the hippocampus. Neuroscience 8, 791-797.

Li, L., Bender, K. J., Drew, P. J., Jadhav, S P., Sylwestrak, E., and Feldman, D. E. (2009). Endocannabinoid signaling is required for development and critical period plasticity of the whisker map in somatosensory cortex. Neuron 64 537-549.

MacMillan, G. J. (1979). An analysis of pigment cell development in the periodic albino mutant of Xenopus. J. Embryol. Exp. Morphol. 52, 165-170.

Magee, J. C., and Johnston, D. (1997) A synaptically controlled, associative signal for Hebbian plasticity in hippocampal neurons. Science 275 209-213.

Markram, H. Lübke, J., Frotscher, M. and Sakman, B. (1997). Regulation of synaptic efficacy by coincidence of postsynaptic APs and EPSPs. Science 275, 213-215.

Mu, Y., and Poo, M. M. (2006). Spike timing-dependent LTP/LTD mediates visual experience-dependent plasticity in a developing retinotectal system. Neuron 50, 115-125.

Nevian T., and Sakmann, B. (2004). Single spine $\mathrm{Ca}^{2+}$ signals evoked by coincident EPSPs and backpropagating action potentials in spiny stellate cells of layer 4 in the juvenile rat somatosensory barrel cortex. J. Neurosci. 24 1689-1699.

Nieuwkoop, P. D., and Faber, J. (1956). Normal Table of Xenopus laevis (Daudin): A Systematical and Chronological Survey of the Development from the Fertilized Egg till the End of Metamorphosis. Amsterdam: North-Holland Pub. Co.

O’Rourke, N.A., and Fraser, S. E. (1990) Dynamic changes in optic fiber terminal arbors lead to retinotopic map formation: an in vivo confocal microscopic study. Neuron 5 159-171.

Pratt, K. G., Dong, W., and Aizenman, C. D. (2008). Development and spike timing-dependent plasticity of recurrent excitation in the Xenopus optic tectum. Nat. Neurosci. 11, 467-475.

Ruthazer, E. S., Akerman, C. J., and Cline, H. T. (2003). Control of axon branch dynamics by correlated activity in vivo. Science 301, 66-70.

Ruthazer, E. S., and Cline, H. T. (2004) Insights into activity-dependent map formation from the retinotectal system: a middle-of-the-brain perspective. J. Neurobiol. 59, 134-146.

Sakaguchi, D. S., and Murphey, R. K. (1985). Map formation in the developing Xenopus retinotectal system: an examination of ganglion cell terminal arborizations. J. Neurosci. 5, 3228-3245.

Schmidt, J. T. (1990). Long-term potentiation and activity-dependent retinotopic sharpening in the regenerating retinotectal projection of goldfish: common sensitive period and sensitivity to NMDA blockers. J. Neurosci. 10, 233-246.

Schwartz, N., Schohl, A., and Ruthazer, E. S. (2009). Neural activity regulates synaptic properties and dendritic structure in vivo through calcineurin/NFAT signaling. Neuron 62, 655-669.

Sjostrom, P. J., Turrigiano, G. G., and Nelson, S. B. (2001). Rate, timing, and cooperativity jointly determine cortical synaptic plasticity. Neuron 32, 1149-1164

Tao, H. W., and Poo, M.-M. (2005). Activity-dependent matching of excitatory and inhibitory inputs during refinement of visual receptive fields. Neuron 45, 829-836.

Tao, H. Z., Zhang, L. I., Engert, F., and Poo, M. (2001). Emergence of input specificity of ltp during development of retinotectal connections in vivo. Neuron 31, 569-580.

Vislay-Meltzer, R. L., Kampff, A. R., and Engert, F. (2005). Spatiotemporal specificity of neuronal activity directs the modification of receptive fields in the developing retinotectal system. Neuron 50, 101-114.

Wiesel, T. (1982). Postnatal development of the visual cortex and the influence of environment. Nature 299 583-591.

Wu, G., Malinow, R., and Cline, H. T. (1996). Maturation of a central glutamatergic synapse. Science 274, 972-976.

Zhang, L. I., Tao, H. W., Holt, C. E., Harris, W.A., and Poo, M.M. (1998).A critical window for cooperation and competition among developing retinotectal synapses. Nature 395, 37-44. 
Zhang, L. I., Tao, H. W., and Poo, M. (2000). Visual input induces longterm potentiation of developing retinotectal synapses. Nat. Neurosci. 3, 708-715.

Zhou, Q., Tao, H. W., and Poo, M. M. (2003) Reversal and stabilization of synaptic modifications in a devel- oping visual system. Science 300, 1953-1957.

Conflict of Interest Statement: The authors declare that the research was conducted in the absence of any commercial or financial relationships that could be construed as a potential conflict of interest.
Received: 01 February 2010; paper pending published: 01 March 2010; accepted: 17 May 2010; published online: 11 June 2010.

Citation: Tsui J, Schwartz N and Ruthazer ES (2010) A developmental sensitiveperiod for spike timing-dependent plasticity in the retinotectal projection. Front. Syn. Neurosci. 2:13. doi: 10.3389/fnsyn.2010.00013
Copyright () 2010 Tsui, Schwartz and Ruthazer. This is an open-access article subject to an exclusive license agreement between the authors and the Frontiers Research Foundation, which permits unrestricted use, distribution, and reproduction in any medium, provided the original authors and source are credited. 